\title{
Electroless Deposition of Cobalt Nanowires in an Aqueous Solution under External Magnetic Field
}

\section{$\operatorname{AUTHOR}(\mathrm{S}):$}

Balela, Mary Donnabelle L.; Yagi, Shunsuke; Matsubara, Eiichiro

\section{CITATION:}

Balela, Mary Donnabelle L....[et al]. Electroless Deposition of Cobalt Nanowires in an Aqueous Solution under External Magnetic Field. ELECTROCHEMICAL AND SOLID STATE LETTERS 2011, 14(6): D68-D71

\section{ISSUE DATE:}

2011

URL:

http://hdl.handle.net/2433/163420

RIGHT:

(C) 2011 ECS - The Electrochemical Society 


\section{Electrochemical and} Solid-State Letters

\section{Electroless Deposition of Cobalt Nanowires in an Aqueous Solution under External Magnetic Field}

Mary Donnabelle L. Balela, Shunsuke Yagi and Eiichiro Matsubara

Electrochem. Solid-State Lett. 2011, Volume 14, Issue 6, Pages D68-D71. doi: $10.1149 / 1.3568829$

Email alerting service
Receive free email alerts when new articles cite this article - sign up in the box at the top right corner of the article or click here

To subscribe to Electrochemical and Solid-State Letters go to:

http://esl.ecsdl.org/subscriptions

(C) 2011 ECS - The Electrochemical Society 


\title{
Electroless Deposition of Cobalt Nanowires in an Aqueous Solution under External Magnetic Field
}

\author{
Mary Donnabelle L. Balela, ${ }^{*, z}$ Shunsuke Yagi, ${ }^{* *}$ and Eiichiro Matsubara \\ Department of Materials Science and Engineering, Kyoto University, Kyoto 606-8501, Japan
}

We report a fast preparation method for cobalt (Co) nanowires of about $59 \mathrm{~nm}$ in diameter and up to $30 \mu \mathrm{m}$ in length by electroless deposition in an aqueous solution at room temperature under an external magnetic field. The reduction process is observed by in situ monitoring of mixed potentials. When the mixed potential decreases below the oxidation-reduction potential of the $\mathrm{Co}$ (II) $/ \mathrm{Co}$ redox pair $\left(E_{\mathrm{Co}(\mathrm{II}) / \mathrm{Co}}\right)$, spherical Co nanoparticles are formed in the solution and then magnetized by the magnetic field. Attractive dipolar interactions are generated along the magnetic field direction, which results in the self-assembly of Co nanoparticles into nanowires.

(c) 2011 The Electrochemical Society. [DOI: 10.1149/1.3568829] All rights reserved.

Manuscript submitted December 7, 2010; revised manuscript received February 7, 2011. Published March 22, 2011.

Magnetic nanomaterials with controllable morphology and dimensions are important for the fabrication of nanoscale devices and functional materials. ${ }^{1,2}$ Recently, the formation of one-dimensional (1-D) nanostructures, such nanowires and nanorods, has attracted considerable attention due to their large anisotropic magnetism and surface area, which are excellent for applications in permanent magnets, high-density magnetic storage media, sensors, and catalysts. 3 - 1 -D Co nanostructures have been prepared by a variety of methods, including microlithography, ${ }^{5}$ vapor-liquidsolid growth, ${ }^{6}$ surfactant-assisted electroless deposition, ${ }^{7-9}$ and template-based electrodeposition. ${ }^{5,10-14}$ Among these methods, template-based electrodeposition has successfully fabricated wellaligned magnetic nanowires and nanorods with controllable diameter and length. However, application of these methods to large-scale production is complicated by the preparation of the templates and their subsequent removal from the nanowires or nanorods. ${ }^{15-17}$ Recently, the application of magnetic fields during solution reduction, e.g. hydrothermal and solvothermal syntheses, has been widely used to fabricate 1-D Co nanostructures due to its simplicity and effectiveness. ${ }^{18-20}$ However, these processes need high temperature, high pressure, and long reaction time.

We have synthesized metallic spherical Co nanoparticles by electroless deposition in an aqueous solution at room temperature using hydrazine monohydrate $\left(\mathrm{N}_{2} \mathrm{H}_{4} \mathrm{H}_{2} \mathrm{O}\right)$ as a reductant. ${ }^{21,22}$ The overall cobalt deposition reactions are described as follows

$$
\begin{gathered}
\mathrm{Co}(\mathrm{II})+1 / 2 \mathrm{~N}_{2} \mathrm{H}_{4}+2 \mathrm{OH}^{-} \rightarrow \mathrm{Co}+1 / 2 \mathrm{~N}_{2}+2 \mathrm{H}_{2} \mathrm{O} \\
\mathrm{Co}(\mathrm{II})+2 \mathrm{~N}_{2} \mathrm{H}_{4}+2 \mathrm{OH}^{-} \mathrm{Co} \rightarrow \mathrm{N}_{2}+2 \mathrm{NH}_{3}+2 \mathrm{H}_{2} \mathrm{O}
\end{gathered}
$$

where $\mathrm{Co}(\mathrm{II})$ represents all $\mathrm{Co}(\mathrm{II})$ species in the solution, e.g. $\mathrm{Co}^{2+}$, $\mathrm{Co}(\mathrm{OH})_{2}, \mathrm{HCoO}_{2}^{-}$or $\mathrm{Co}(\mathrm{OH})_{3}^{-}, \mathrm{CoO}_{2}^{2-}$ and $\mathrm{Co}(\mathrm{OH})_{4}^{2-}$. In this work, Co nanowires are fabricated by performing the above reactions under a magnetic field of $10 \mathrm{~T}$. The formation mechanism of Co nanowires is investigated by observing the change in mixed potential with time and the evolution of morphology and structure of the products under a magnetic field. The present method offers a simple and inexpensive room-temperature fabrication for Co nanowires in an aqueous solution.

\section{Experimental}

All chemical reagents used were reagent-grade (Nacalai Tesque, Inc.). The $\mathrm{Co}$ (II) solution was prepared by dissolving $0.010 \mathrm{~mol}$ $\mathrm{Co}$ (II) acetate tetrahydrate $\left(\mathrm{Co}\left(\mathrm{C}_{2} \mathrm{H}_{3} \mathrm{O}_{2}\right)_{2} \cdot 4 \mathrm{H}_{2} \mathrm{O}\right), 1.3 \times 10^{-4} \mathrm{~mol}$ poly(ethylene glycol) $\left(\mathrm{PEG}, \mathrm{H}\left(\mathrm{OCH}_{2} \mathrm{CH}_{2}\right) \mathrm{nOH}, M_{\mathrm{w}}=20,000\right)$, and $0.025 \mathrm{~mol} \mathrm{NaOH}$ in $75 \mathrm{~cm}^{-3}$ distilled water while nitrogen gas $\left(\mathrm{N}_{2}\right)$ was bubbled at $50 \mathrm{~cm}^{-3} \mathrm{~min}^{-1}$. Then, $2.5 \times 10^{-5}$ mol chloro-

\footnotetext{
* Electrochemical Society Student Member.

** Electrochemical Society Active Member.

${ }^{\mathrm{z}}$ E-mail: balela.m@hw8.ecs.kyoto-u.ac.jp
}

platinic acid hexahydrate $\left(\mathrm{H}_{2} \mathrm{PtCl}_{6} \cdot 6 \mathrm{H}_{2} \mathrm{O}\right)$ in $10 \mathrm{~cm}^{-3}$ propylene glycol $\left(\mathrm{PG}, \mathrm{C}_{3} \mathrm{H}_{8} \mathrm{O}_{2}\right)$ solution was added as a nucleating agent. The reducing agent solution was then prepared by dissolving $0.10 \mathrm{~mol}$ $\left(5 \mathrm{~cm}^{-3}\right) \mathrm{N}_{2} \mathrm{H}_{4} \mathrm{H}_{2} \mathrm{O}$ in $25 \mathrm{~cm}^{-3}$ deaerated PG. The Co(II) solution was placed under $10 \mathrm{~T}$ immediately after adding the $\mathrm{N}_{2} \mathrm{H}_{4}$ solution. The final solution had a total volume of $115 \mathrm{~cm}^{-3}$ and the final concentrations of the reagents were $0.087 \mathrm{M} \mathrm{Co}(\mathrm{II})$ acetate, $1.1 \mathrm{mM}$ PEG, $0.22 \mathrm{M} \mathrm{NaOH}, 0.22 \mathrm{mM} \mathrm{H}_{2} \mathrm{PtCl}_{6}$, and $0.87 \mathrm{M} \mathrm{N}_{2} \mathrm{H}_{4}$. After the reaction for $1 \mathrm{~h}$, Co nanowires were magnetically separated from the solution and ultrasonicated for $10 \mathrm{~min}$ in ethanol $\left(\mathrm{C}_{2} \mathrm{H}_{6} \mathrm{O}\right)$. This washing process was repeated 10 times to remove organic contaminants.

The samples were observed by a field-emission-scanning electron microscope (JEOL JSM 6500-F) and a transmission electron microscope (TEM, JEOL JEM 2010). The mean diameter was calculated from the values of 100 nanowires measured by image analysis. Similarly, the lengths of the nanowires were also measured. The crystalline structure was analyzed by X-ray diffraction (XRD, MAC Science M03XHF22) using Cr $K \alpha$ radiation. The mixed potential was monitored by a potentiostat/galvanostat (Hokuto Denko Co. Ltd., HA-151) using a Ptsputtered quartz crystal substrate and a silver/silver chloride $(\mathrm{Ag} / \mathrm{AgCl})$ electrode in $3.33 \mathrm{M}$ potassium chloride $(\mathrm{KCl})$ aqueous solution (Horiba 2565-10T) as working and reference electrodes, respectively.

\section{Results and Discussion}

Figure 1 shows the electron micrographs of the products after electroless deposition in an aqueous solution at room temperature under 0 and $10 \mathrm{~T}$. Spherical Co nanoparticles with a mean diameter of about $54 \mathrm{~nm}$ are prepared under $0 \mathrm{~T}$ as shown in Fig. 1a. When a magnetic field of $10 \mathrm{~T}$ is applied, Co nanowires of less than $30 \mu \mathrm{m}$ long and an average diameter of about $59 \mathrm{~nm}$ are formed. The nanowires in Fig. 1b consist of self-aligned spherical nanoparticles. Although Co nanoparticles in Fig. 1a are somewhat linearly agglomerated even without the magnetic field, the morphology of the nanowires fabricated under $10 \mathrm{~T}$ is evidently different from them. Further, the nanowires exhibit some degree of mechanical strength since they maintain the morphology even after ultrasonication during washing. Without a magnetic field, Co nanoparticles magnetize one another by weak dipolar interactions, resulting in agglomeration during the reaction. In contrast, the Co nanoparticles are strongly magnetized by an external magnetic field, and localized magnetic fields are induced along the field direction. ${ }^{17}$ Consequently, the dipole moments of Co nanoparticles are increased and self-assembly of Co nanoparticles into nanowires proceeds parallel to the magnetic field.

To elucidate the influence of magnetic fields on the assembly of Co nanoparticles in an aqueous solution, $52 \mathrm{~nm}$ Co nanoparticles are subjected to a field of $10 \mathrm{~T}$ for $1 \mathrm{~h}$ after washing. As shown in 
(a) $0 \mathrm{~T}$

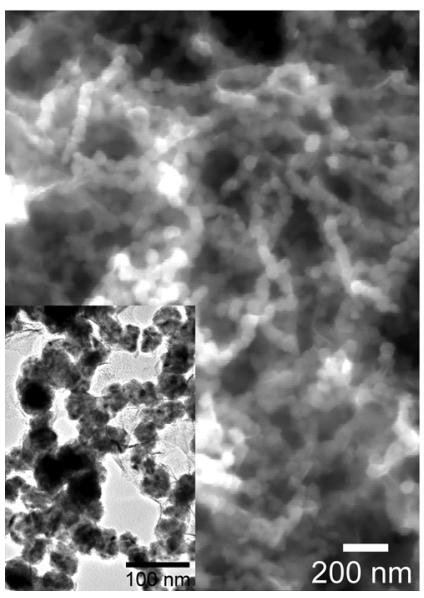

(b) $10 \mathrm{~T}$

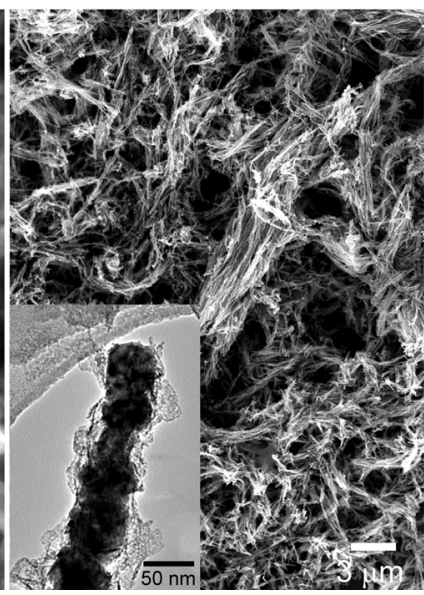

(c) $10 \mathrm{~T}$, post-synthesis

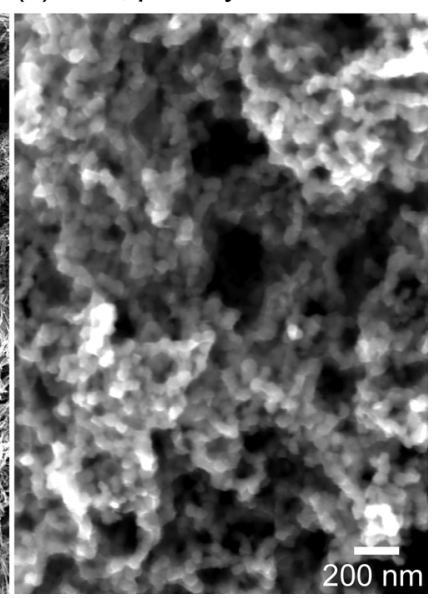

Figure 1. SEM and TEM images of products obtained by electroless deposition of Co in an aqueous solution at room temperature under (a) 0 and (b) 10 T. (c) SEM image of $52 \mathrm{~nm}$ Co nanoparticles subjected to a magnetic field of $10 \mathrm{~T}$ for $1 \mathrm{~h}$ after washing.
Fig. 1c, only interlinked Co nanoparticles are present in the solution and nanowires are not formed. Since Co nanoparticles could be magnetized by strong magnetic fields, it is reasonable to expect that the nanoparticles would also assemble into linear chains. However, attractive dipole interaction between Co nanoparticles is possibly too weak to maintain the linear chains. They easily break up during washing unless Co atoms, which are generated in the solution during electroless deposition, deposit on the gaps between nanoparticles and fix the structure.

Figure 2 shows the corresponding XRD profiles of the samples. The diffraction peaks of the products are fundamentally attributed to both hexagonal close-packed (hcp) and face-centered cubic (fcc) Co. The strongest peak at $68.0^{\circ}$ and the small peak at $80.5^{\circ}$ are 111 and 200 of fcc Co, respectively. The peak at $68.0^{\circ}$ has a shoulder at a higher scattering angle because of the presence of the $002 \mathrm{hcp}$ Co peak at $68.9^{\circ}$. The diffused peaks at $63.8^{\circ}$ and $73.6^{\circ}$ are 100 and 101 of hcp Co. The crystallite sizes of Co nanoparticles $(12 \mathrm{~nm})$ and nanowires $(15 \mathrm{~nm})$ calculated from the $111 \mathrm{fcc}$ Co peaks by Scherrer's equation are smaller than the apparent particle sizes or the apparent wire diameters $(\sim 54-59 \mathrm{~nm})$. This indicates that they are polycrystals. A very weak peak at about $55.5^{\circ}$ is attributed to $\mathrm{CoO}$, which is formed on the surface of the products during washing and XRD measurements in air after the reaction.

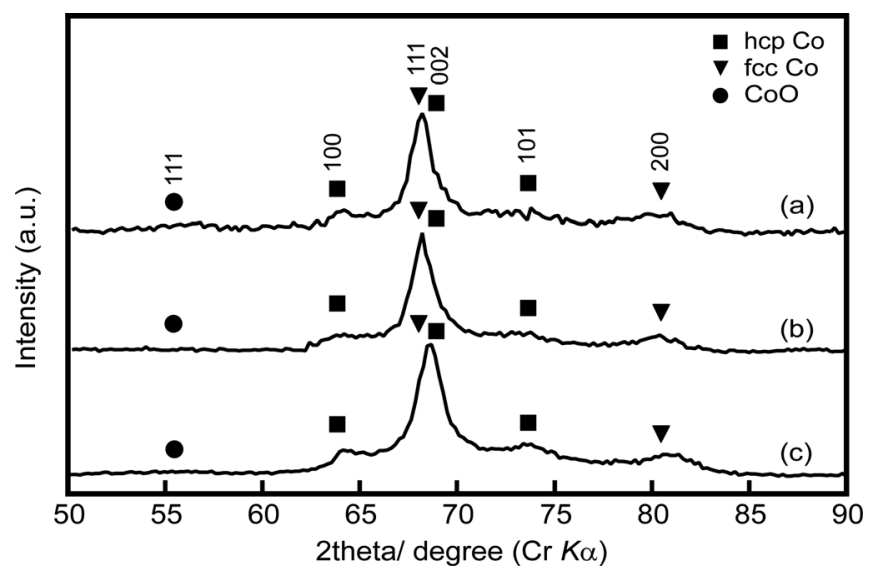

Figure 2. XRD profiles of Co products prepared by electroless deposition in an aqueous solution at room temperature under (a) 0 and (b) 10 T. (c) Corresponding XRD pattern of $52 \mathrm{~nm}$ Co nanoparticles subjected to a magnetic field of $10 \mathrm{~T}$ for $1 \mathrm{~h}$ after washing.
The fabrication process of Co nanowires under the magnetic field is investigated by in situ mixed potential measurement and by sampling products during the reaction. Figures $3 a-3 d$ show SEM images of precipitates obtained after 5, 10, 15, and $20 \mathrm{~min}$. At the start of the reaction (5-10 min), spherical Co nanoparticles are observed and show no clear difference regardless of the applied magnetic field. The nanoparticles slightly grow from 44 to $52 \mathrm{~nm}$ from 5 to $10 \mathrm{~min}$. Short chain-like nanowires up to $1 \mu \mathrm{m}$ long are formed by $15 \mathrm{~min}$. The nanowires have a mean diameter of about $52 \mathrm{~nm}$, which is identical to the size of the Co nanoparticles obtained after $10 \mathrm{~min}$. This suggests that self-assembly of Co nanoparticles must have started after they have grown to a certain size of about $52 \mathrm{~nm}$. Co nanowires grow up to $20 \mu \mathrm{m}$ long after $20 \mathrm{~min}$ under $10 \mathrm{~T}$. Further increase in reaction time results in longer Co nanowires with slightly larger diameters. On the other hand, the corresponding XRD patterns of the products obtained under $10 \mathrm{~T}$ (Fig. 3e) show the same hcp and fcc Co peaks with similar intensity ratios. This suggests that the structure of Co is not affected by the magnetic field.

Figure 4 shows the changes in mixed potential with time during electroless deposition of Co. From the thermodynamical viewpoint, Co can only be deposited when the mixed potential is less than the redox potential of the $\mathrm{Co}(\mathrm{II}) / \mathrm{Co}$ redox couple, $E_{\mathrm{Co}(\mathrm{II}) / \mathrm{Co}}=-0.62 \mathrm{~V}$ vs SHE (standard hydrogen electrode), calculated by the Nernst equation assuming that the $\mathrm{Co}^{2+}$ aquo ions equilibrate with $\mathrm{Co}(\mathrm{OH})_{2}$ and the total concentration of $\mathrm{Co}(\mathrm{II})$ is $0.087 \mathrm{M}^{21-23}$ The mixed potential decreases below $-0.62 \mathrm{~V}$ after $4 \mathrm{~min}$ and the solution color starts changing from pink to black. This indicates that a very fast reduction of $\mathrm{Co}$ (II) has started, i.e. many Co atoms are generated at a time in the solution. This is consistent with the SEM and XRD analyses of the product, i.e. the $42 \mathrm{~nm}$ Co nanoparticles in Fig. 3a is metallic Co (Fig. 3e). At $10 \mathrm{~min}$, the mixed potential decreases to about $-0.78 \mathrm{~V}$, which implies larger driving force for $\mathrm{Co}$ (II) reduction. $\mathrm{Co}$ (II) ions are then more easily reduced and the diameter of the nanoparticles is increased as shown in Fig. 3b. The Co nanoparticles rapidly grow in all lattice directions even under the magnetic field, which coincide with the XRD profiles in Fig. 3e. The mixed potential then increases gradually after $10 \mathrm{~min}$ since the total anodic current progressively decreases as more hydrazine is consumed by the reduction reactions. $^{21,22}$

A schematic diagram illustrating the stepwise formation of $\mathrm{Co}$ nanowires in aqueous solution under an external magnetic field is shown in Fig. 5. At the early stage (Step I), minute Co nanoparticles are formed in the solution as primary particles, regardless of the presence of an external magnetic field as in Fig. 3a. Generally, the orientation of each nanoparticle in the solution is random when no 
(a) 5 min

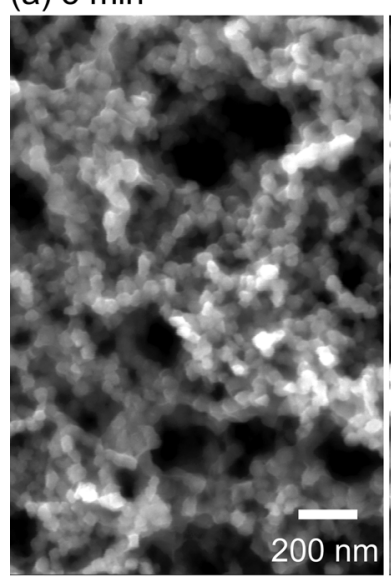

(b) $10 \mathrm{~min}$

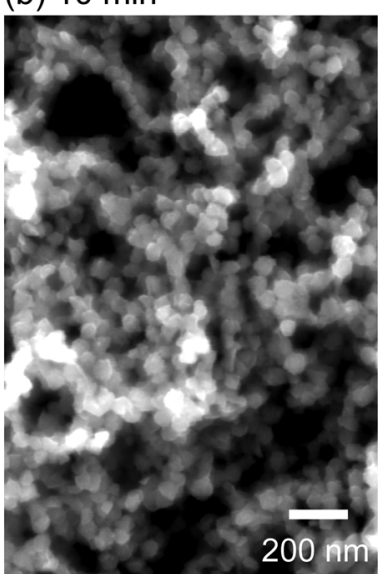

(d) $20 \mathrm{~min}$

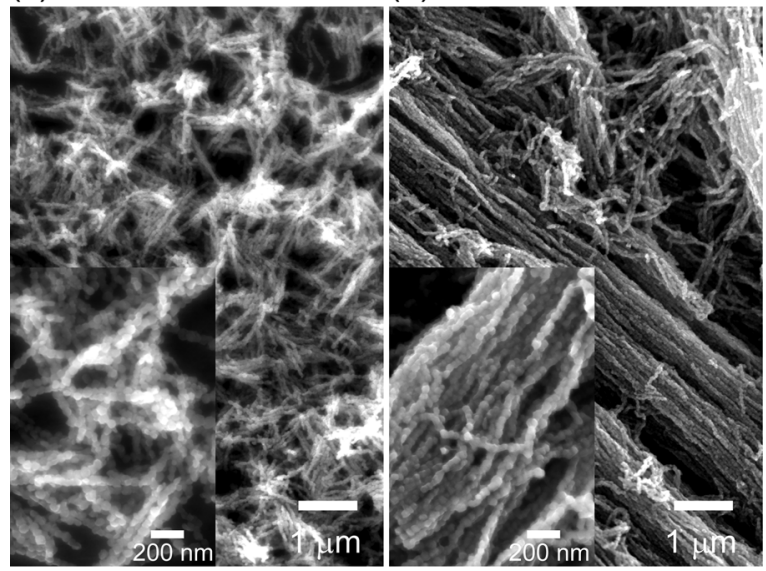

(e) XRD profiles of products

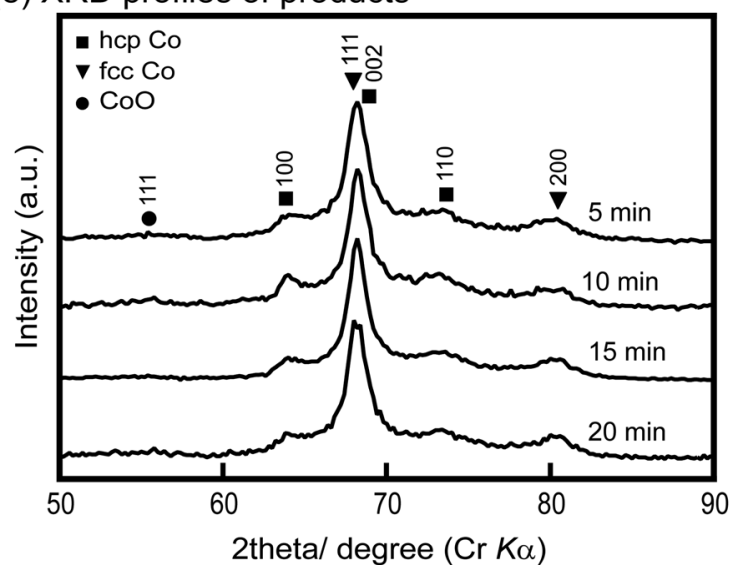

Figure 3. SEM images of the products obtained during electroless deposition in an aqueous solution at room temperature under $10 \mathrm{~T}$ at reaction times of (a) 5, (b) 10, (c) 15, and (d) $20 \mathrm{~min}$. (e) Corresponding XRD profiles of the products fabricated at different reaction times under $10 \mathrm{~T}$.

external field is applied. In the presence of a strong magnetic field, these nanoparticles are magnetized and would start to align. Since the dipole magnetic moment is proportional to the volume of a nanoparticle, the interaction between the nanoparticles is too weak to overcome solvent motion during the reaction. With a longer reaction time, the primary Co nanoparticles grow (Fig. 3b) as in Stage II and their magnetic interaction becomes stronger to be significantly affected by the magnetic field. Subsequently, when the dipole magnetic moments of the nanoparticles are oriented along the external

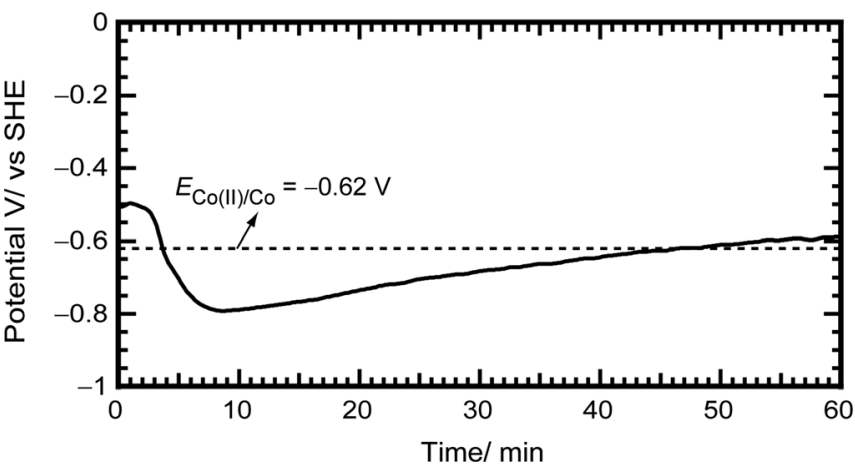

Figure 4. Change in mixed potentials with time during electroless deposition of Co nanowires in an aqueous solution at room temperature under $10 \mathrm{~T}$.

magnetic field, attractive interactions are induced parallel to the magnetic field. Thus, neighboring Co nanoparticles start to assemble along the magnetic field direction and form linear nanoparticle chains (Stage III) as shown in Fig. 3c. As the reaction proceeds, Co atoms, which are simultaneously generated in the solution, deposit especially on the gaps between the spherical nanoparticles because of the reduction of interfacial energy as in Stage III. This leads to the formation of $\mathrm{Co}$ nanowires. If a stronger magnetic field is applied, formation of thick parallel arrays of Co nanowires occurs as in Fig. 3d (Stage IV).

\section{Conclusion}

Co nanowires of about $59 \mathrm{~nm}$ diameter and lengths up to 30 $\mu \mathrm{m}$ are prepared by electroless deposition in an aqueous solution at room temperature under an external magnetic field. When the mixed potential of the solution decreases below $E_{\mathrm{Co}(\mathrm{II}) / \mathrm{Co}}$, spherical Co nanoparticles are first precipitated in the solution, regardless of the magnetic field. The Co nanoparticles grow and then orientate parallel to the magnetic field. These oriented Co nanoparticles attach to one another due to attractive dipolar interactions, and $\mathrm{Co}$ atom deposition on the gaps between the nanoparticles stabilizes the structure. Subsequently, strong Co nanowires are formed.
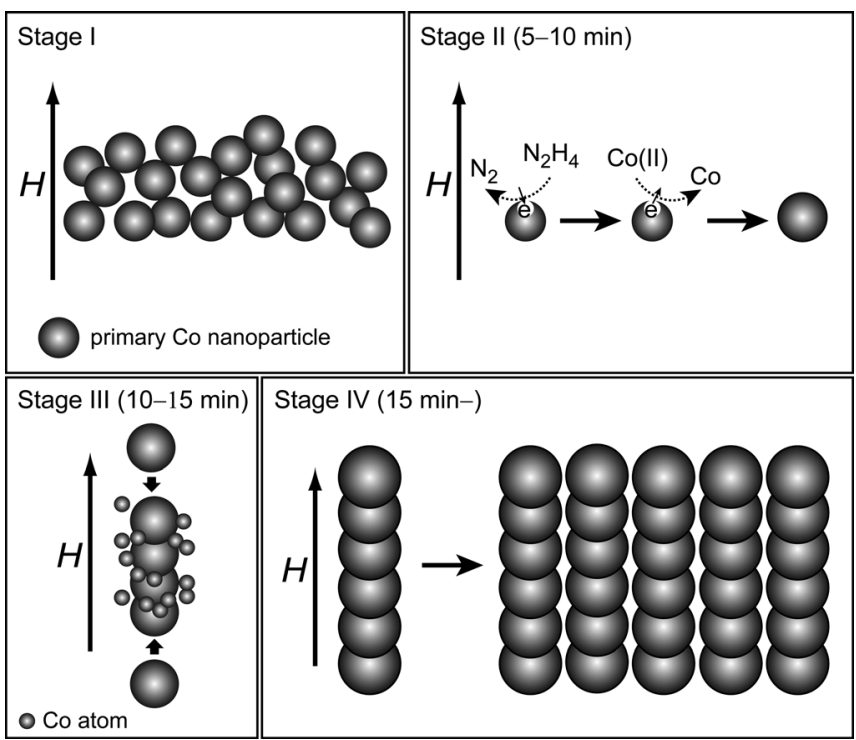

Figure 5. Schematic diagram of the formation of Co nanowires by electroless deposition in an aqueous solution at room temperature under an external magnetic field 


\section{Acknowledgments}

The authors express their gratitude to Associate Professor Tetsu Ichitsubo, Ph.D. and Kenji Kazumi from Kyoto University for the assistance during experiment. This research was supported by a grantin-aid for Knowledge Cluster Initiative (Kyoto Nanotechnology Cluster), grant-in-aid for the Global COE Program (International Center for Integrated Research and Advanced Education in Materials Science), and grant-in-aid for Young Scientists (B20760505), all from the Japan Society for the Promotion of Science, Ministry of Education, Culture, Sports, Science and Technology of Japan.

Kyoto University assisted in meeting the publication costs of this article.

\section{References}

1. S. Yagi, M. Kawamori, and E. Matsubara, Electrochem. Solid. State Lett., 13, E13 (2010).

2. G. Zhang, T. Zhang, X. Lu, W. Wang, J. Qu, and X. Li, J. Phys. Chem., 111, 12663 (2007)

3. G. Viau, C. Garcia, T. Maurer, G. Chaboussant, F. Ott, Y. Soumare, and J.-Y. Piquemal, Phys. Status Solidi. A, 206, 6636 (2009).

4. B.-Y. Xie, Y. Qian, S. Zhang, S. Fu, and W. Yu, Eur. J. Inorg. Chem., 2006, 2454 (2006).

5. M. Aslam, R. Bhobe, N. Alem, S. Donthu, and V. P. Dravid, J. Appl. Phys., 98, $074311(2005)$
6. E. K. Athanassiou, P. Grossman, R. N. Grass, and W. J. Stark, Nanotechnology, 18, 165606 (2007).

7. Y. Soumare, J.-Y. Piquemal, T. Maurer, F. Ott, G. Chaboussan, A. Falqui, and G. Viau, J. Mater. Chem., 18, 5696 (2008)

8. S. I. Cha, C. B. Mo, K. T. Kim, and S. H. Hong, J. Mater. Res., 20, 2148 (2005).

9. Y. Li, J. Zhao, Y. Zhu, D. Ma, Y. Zhao, S. Hou, and F. Yan, Colloids Surf., A 356, 156 (2010).

10. K. Hoshino and Y. Hitsuoka, Electrochem. Comm., 7, 821 (2005).

11. N. B. Chaure, P. Stamenov, F. M. F. Rhen, and J. M. D. Coey, J. Magn. Magn. Mater., 290-291, 1210 (2005).

12. X. Yuan, C. Du, G. Sun, and N. Pan, Appl. Surf. Sci., 253, 4546 (2007).

13. L. Vila, P. Vincent, L. Dauginet-De Pra, G. Pino, E., Minoux, L., Gangloff, S. Demoustier-Champagne, N. Sarazin, E. Ferain, R. Legras, Nano Lett., 4, 521 (2004).

14. A. Kazadi Mukenga Bantu, J. Rivas, G. Zaragoza, M. A. Lopez-Quintela, and M. C. Blanco, J. Appl. Phys., 89, 3393 (2001).

15. J. Wang, M. Yao, C. Xu, Y. Zhu, and P. Cui, Mater. Lett, 62, 3431 (2008).

16. C. Gong, J. Tian, Z. Wu, and Z. Zhang, Mater. Res. Bull., 44, 35 (2009).

17. C. Gong, L. Yu, Y. Duan, J. Tian, Z. Wu, and Z. Zhang, Eur. J. Inorg. Chem., 2008, 2884 (2008).

18. H. Niu, Q. Chen, H. Zhu, Y. Lin, and X. Zhang, J. Mater. Chem., 13, 1803 (2003).

19. J. Park, Y. Jun, J. Choi, and J. Cheon, Chem. Commun., 2007, 5001 (2007).

20. M. J. Hu, B. Lin, and S. H. Yu, Nano. Res., 1, 303 (2008).

21. M. D. L. Balela, S. Yagi, and E. Matsubara, Electrochem. Solid. State Lett., 13, D4 (2010).

22. M. D. L. Balela, S. Yagi, and E. Matsubara, ECS Trans., 28(7), 29 (2010).

23. S. Yagi, H. Nakanishi, T. Ichitsubo, and E. Matsubara, J. Electrochem. Soc., 156, D321 (2009). 